\title{
Prostate cancer stem cells
}

\author{
Hanna Romańska-Knight ${ }^{1}$ and Paul Abe/ ${ }^{2}$
}

'Department of Molecular Pathology and Neuropathology, Medical University of Łódź, Poland

2Department of Surgery and Cancer, Imperial College London, Hammersmith Campus, London, Great Britain

\section{KEY WORDS}

prostate cancer stem cell D cancer stem cell

\section{ABSTRACT}

The Cancer Stem Cells (CSCs) hypothesis postulates that a minute subpopulation of cells is accountable for cancer initiation and progression. Unlike the stochastic and clonal evolution models, the CSC theory proposes that tumours are hierarchical and only the rare subset of cells at the top of the 'stemness hierarchy tree' are adequately 'equipped' biologically to initiate and drive tumourigenesis. CSCs have been implicated in various solid malignancies including prostate cancer ( $\mathrm{PCa}$ ) where their existence seems to provide an explanation for the failure of tumour eradicating therapies. As CSCs are thought to share many properties with normal stem cells, understanding normal stem cells should shed light on the pathomechanisms of cancer and, importantly, on potential therapeutic interventions. The purpose of this paper is to review the existing data on CSCs in $\mathrm{PCa}$, their putative phenotypic markers, potential role in tumour biology and relevance to therapy.

\section{INTRODUCTION}

The hypothesis of Cancer Stem Cells (CSCs) dictates that a small subpopulation of undifferentiated, self-renewing cells found in most, if not all, cancers is responsible for tumour initiation and sustained growth $[1,2]$. CSCs are thought to originate from the stem cell compartments of normal organs and to share many properties with normal stem cells. They are also believed to be accountable for the failure of eradicating therapies $[1,2]$. Therefore, an understanding of their biology should have significant clinical benefits. In particular, in prostate cancer (PCa), identification of PCa stem cells (PCSCs) holds promise to combat castration-resistant (CR) PCa, a major clinical challenge of contemporary urology.

A series of excellent reviews discuss a potential role of CSCs in development of various human cancers [3-6]. Here, we provide a concise updated summary of existing literature on CSC biology, putative markers and clinical relevance of CSCs in solid cancers with an emphasis on PCa.

\section{Stem cells}

Stem cells are integral components of normal mammalian physiology. They differ in origin, location, proliferative potential and the range of cell lineages they are able to produce. All stem cells, regardless of their source, have two cardinal attributes: 1) longevity (the ability of indefinite self-renewal) and 2) multipotency (a capacity to give rise to progenitors and differentiated progenies) [7]. The 'level' of potency decides on the position of the cell in the 'stemness hierarchy', where the supremacy resides in the zygote, the ultimate totipotent stem cell.

Multipotency and self-renewal are dependent on a number of determinants including transcription factors such as 0ct4, Sox2 and Nanog, Bmi-1 oncogene, as well as activation of telomerase and several signalling pathways, in particular, $\mathrm{PI} 3 \mathrm{~K}, \mathrm{Wnt}$, and Notch [8-14].

Adult (or tissue) stem cells are believed to exist in almost all post-embryonic tissues. They reside in a specialized microenvironment, a so-called niche. This provides a physical anchor and, through a combination of direct and indirect interactions between neighboring cells and extracellular matrix (ECM) components, enables a balance between quiescence and self-renewal that supports stem cell identity. Attachment of a stem cell to the niche ensures that daughter cells leave the niche and move into new microenvironments where, being exposed to different extrinsic signals, they lose 'stemness' and commit to cell lineages specific to the tissue in which they reside [15]. Under physiological conditions a stem cell pool is important for maintaining the integrity of the tissue. Stem cells are also a key element in normal tissue regeneration and repair.

\section{The normal prostate}

The double-layered epithelium of the human adult prostate makes up glandular structures, which are comprised of three principal types of cells identified by their morphological appearance, location, and phenotypic profile. Luminal cells $\left.\left(C K 8^{+} / C K 18^{+} / \mathrm{AR}^{+} / \mathrm{PSA}^{+} / \mathrm{PAP}\right)^{+}\right)$, the main component of the lining of the glands, are terminally differentiated and phenotypically express cytokeratins 8 and 18, androgen receptor (AR), prostate specific antigen (PSA), and prostatic acid phosphatase (PAP). Their maintenance and function are androgen-dependent. Basal cells $\left(\mathrm{CK}^{+} / \mathrm{CK} 14^{+} / \mathrm{p63} 3^{+} / C D 44^{+} / A R^{-}\right)$comprise less than $10 \%$ of the total epithelium, are relatively undifferentiated, and phenotypically express cytokeratins 5 and 14, p63, and CD44, but neither PAP nor PAS [16]. Expression of AR is low or undetectable and the cells are androgen-responsive, but not dependent. Neuroendocrine cells $\left(A R^{-}\right)$, the least frequent type, are terminally differentiated. They contain serotonin, calcitonin, and somatostatin, do not express $A R$, and are not dependent on androgen for their survival [17].

Normal prostate development and cytodifferentiation are critically dependent on complex epithelial-mesenchymal interactions and hormonal stimuli. An inductive role of prostatic stroma in epithelial differentiation has been elegantly demonstrated by a series of in vivo recombinant studies [18-20].

Stem cells in normal prostate

Evidence for the existence of a stem cell compartment in the normal mammalian prostate is based on experimental evidence in mice demonstrating that the adult murine prostate can undergo multiple rounds of castration-induced regression and androgeninduced regeneration [21]. As androgen ablation does not affect basal cells, it has been hypothesized that regenerative capacity, 
that is the compartment containing cells with stem-like characteristics, must reside within the basal layer. This assumption is supported by a number of studies demonstrating not only that p63 (one of the markers of basal cells) is required for prostate organogenesis and development, but also that luminal secretory cells result from the differentiation of p63-expressing basal cells [22]. Location of the stem cell compartment close to the stroma fits the concept of a tissue-specific niche, which through its paracrine activity maintains identity of stem cells and their principal function of tissue regeneration. However, there are several reports of the existence of a subset of $\mathrm{CR}$ and hence androgen-independent cells with stem-like properties within the luminal compartment of the gland as well $[21,23]$. Moreover, results of tissue recombination and grafting experiments by Kurita et al (2004) have shown that embryonic mesenchyme of urogenital sinus from p63-t- mice can regenerate prostate ductal epithelium which consists solely of cells with luminal phenotype [24]. This would suggest that precursors other than basal $p 63^{+}$cells do exist in the prostate and can give rise to luminal cells.

In the adult human prostate, a number of candidate phenotypic markers of stem cells have been proposed and several populations of putative stem cells reported. In particular, cells expressing surface molecules such as CD44 [16], integrin $\alpha_{2} \beta$ and CD133 isolated from normal prostate epithelium have been shown to possess a capacity to differentiate into prostate-specific lineages and to have high proliferative and morphogenic potentials. This small population of presumed prostate stem/progenitor cells $\left(\mathrm{CD}_{4} 4^{+} / \mathrm{CD} 133^{+} / \alpha_{2} \beta 1^{+}\right)$constitute approximately $1 \%$ of the basal layer, where they reside on the basement membrane, anchored by integrin $\alpha_{2} \beta_{1}[25,26]$. They are morphologically intermediate between basal and luminal cells. Both localization and phenotype of these cells give support to their putative stem-like features and, therefore, to the developmental link and hierarchical relationship between the basal and luminal cells.

The most important characteristic of a true stem cell lies in its multipotency, i.e., an ability to recreate in vivo, from a single cell, a fully functional tissue, appropriate to its origin. Moreover, this property has to be maintained during successive transplantations. Despite an extensive worldwide search, the existence of stem cell pools in human peripheral organs remains yet to be conclusively proven due to significant technical difficulties and the limitations of available experimental models.

\section{Cancer stem cells}

The concept that neoplasia is a disease of differentiation is not new. First connections between tissue development and tumour formation are associated with the work of the $19^{\text {th }}$ century German pathologists Rudolph Virchow, Julius Cohnheim and Wilhelm Waldeyer. These advocates of the 'blocked ontogeny' theory postulated that tumour cells must come from normal embryonic cells 'left behind' in the adult organism and that carcinogenesis is closely linked to the arrest and/or distortion of a normal differentiation process [27]. It is claimed that tissue stem cells are the most likely targets of genetic instability as it is they alone whose life span is sufficiently long to acquire the necessary number of genetic changes required for malignant transformation [28]. A survival advantage for stem cells over differentiated cells, combined with clonal selection of cells maintaining stem-like properties and the ability to self- renew, converts normal cell renewal pathways into an engine of neoplastic proliferation. This concept seems to be true and applicable for teratocarcinomas, hematological malignancies and many types of carcinoma.

Due to the potential relevance of CSCs to cancer therapy, recent decades have witnessed an unprecedented revitaliza- tion of the CSC hypothesis. An extensive search for CSCs, or more appropriately, tumour-initiating cells (TICS), has provided evidence to support the original hypothesis and to suggest that their biology is governed by molecular pathways known to be responsible for self-renewal of normal stem cells [29-35]. Thus considerable efforts are being made in the search for a fingerprint of 'stemness' common to normal stem cells and their malignant counterparts. Substantial data have been generated by gene and protein expression analyses resulting in a number of phenotypic markers reflecting a molecular definition of 'stemness'. Broadly, these include molecules involved in: i) maintaining pluripotency (e.g., transcription factors such as 0ct 4, Sox2 and Nanog), ii) selfrenewal (e.g., Bmi1), iii) cytoprotection (e.g., $A B C$ transporters and ALDH, a detoxifying enzyme for alkylating agents such as cyclophosphamide) and iv) adhesion to the niche [8-10, 12, 36-38]. This list is not exhaustive and the panels of proposed markers vary between CSC systems.

\section{Prostate cancer}

$\mathrm{PCa}$ is the commonest cancer in men and second commonest cause of cancer-related death. The incidence of PCa has gradually increased worldwide since the 1960's. In Western Europe over 100 000 men are diagnosed with PCa and 35000 die annually [39]. Primary PC is predominantly of luminal phenotype and hence $\mathrm{AR}^{+}$ and androgen-dependent (AD), so that eliminating or reducing endogenous androgens by surgical or chemical castration results in subjective and objective improvement in approximately 80\% of patients. However, this benefit lasts only for a median period of $\sim 2$ years in men with metastatic bone disease after which there is relapse and death ensues within 6-12 months [40]. Neither the pathogenesis of PCa nor the mechanisms underlying disease progression are fully elucidated. There is therefore a need to further the understanding of the origins of $\mathrm{PCa}$ at a cellular level and to evaluate the potential contribution of stem cells.

\section{Prostate cancer stem cells}

That PCa regrowth occurs even with castrate levels of testosterone points to the existence of a distinct stem-like population of CR cells with tumour-initiating capacity. Multiple cell types have been proposed as the true initiators of human PCa. As cells displaying basal phenotype are frequently found in metastases [41], it was proposed that to survive and expand in an androgen-deprived environment and not to express AR, PCa-initiating cells ought to be phenotypically identical to the normal prostate basal stem-like cell i.e., $\mathrm{CK}^{+} / \mathrm{CK} 14^{+} / \mathrm{AR}-/ \mathrm{p} 63^{+} / \alpha_{2} \beta_{1}{ }^{\text {hi }} / \mathrm{CD} 133^{+} / \mathrm{CD}_{4} 4^{+}[25,26,41]$ Several lines of evidence support this hypothesis. For example, as demonstrated by clonogenic assays, only cells expressing putative PCSC markers had self-renewal potential in vitro [42]. Furthermore, under stimuli inducing cell differentiation in vitro, these supposedly primitive cells gave rise to progenies phenotypically consistent with human differentiated prostatic luminal cells $\left(\mathrm{CK}_{18}{ }^{+} / \mathrm{AR}^{++} / \mathrm{PAP}+\right)$ [42]. Subsequent study by Patrawala and co-workers (2006) showed that a population of highly purified $\mathrm{CD} 44^{+}$cells from xenograft human PCas were enriched in tumourigenic and metastatic progenitors. Indeed, CD44+ PCa cells have been shown to have increased tumourigenic potential in vivo as compared to their CD44- counterparts [43]. Gu and co-workers (2007) reported that clonally-derived PCa cells transfected with human telomerase reverse transcriptase (hTERT) were able to reconstitute the original tumour in vivo [44]. Consistent with the stem cell characteristics, hTERT ${ }^{+}$PCa cells expressed both early progenitors (CD44 and Nestin) and embryonic stem cell markers (Oct4, Sox2 and Nanog) and were AR- and p63-. More recently, Birnie et al, using gene expression profiling, demonstrated that the fusion gene TMPRSS2-ERG, frequently found in CR 
aggressive PCa and associated with PSA failure, was expressed in $\alpha_{2} \beta_{1}{ }^{\text {hi }} / \mathrm{CD} 133^{+}$prostate epithelial cells [45].

Although substantial evidence supports the basal stem-like cell as the main 'PCa-initiator', several studies in rodents have shown that PCa may also originate from the luminal cell layer of the prostatic epithelium. For example, Wang and co-workers (2009) identified in murine prostate a rare luminal cell subset of CR NKx3.1-expressing cells (CARNs) that display stem cell properties. Targeted deletion of the Pten suppressor gene in these cells led to development of $\mathrm{PCa}$, closely mimicking disease progression in man [46]. Although the existence of multiple stem cells could be species-specific reflecting distinctive anatomy of the multilobular murine gland, it is likely that, the luminal cell layer in the human prostate also houses a distinct non-overlapping stem cell population, oncogenic transformation of which may give rise to the development of CR disease [47]. Demonstration that luminal epithelial stem-like cells might be efficient targets of tumour-initiating activating mutations would be in agreement with a commonly accepted model of $\mathrm{PCa}$ development into a tumour of a predominantly luminal phenotype $\left(\mathrm{CK}^{+} \mathrm{CK}_{18}{ }^{+} / \mathrm{AR}^{+} / \mathrm{PSA}^{+} / \mathrm{PAP}^{+}\right)$[48]. However, even if several populations of cells capable of cancer initiation exist in the prostate, the 'stemness lineage hierarchy' seen in the normal gland appears also to be maintained in PCa and the basal stem-like cells are by far the most efficient targets of oncogenic stimuli resulting in neoplastic transformation. Recent functional studies provide support to this hypothesis demonstrating that activation of oncogenic pathways in human prostate basal cells leads to recapitulation of tumour initiation and progression in immunodeficient mice that histologically closely mimics human $\mathrm{PCa}$, with expansion of luminal and concomitant loss of basal cells $[49,50]$.

It is widely accepted that the development of carcinoma is due to accumulation of somatic mutations in epithelial cells supported by the permissive tumour microenvironment. In prostate, recent studies have revealed that ECM, fibroblasts, blood vasculature and inflammatory cells have significant impact on transition from pre-invasive to invasive growth of PCa and should be regarded as crucial and active participants in tumourigenesis. Tumour stroma is recognized as a source of paracrine growth factors such as frizzled-related protein 1 (SFRP1), TGF $\beta 1$, and stromal cell-derived factor 1 (SDF-1/CXCL12) that maintain and potentiate survival and growth of the tumour [51-54]. Signalling via the SDF-1chemokine receptor type 4 (CXCR4) axis leading to activation of anti-apoptotic pathways and stimulation of cell motility and invasion is known to regulate the metastatic behaviour of tumour cells [55]. Interestingly, the receptor for SDF-1 (SDFR-1, or neuroplastin) is expressed by putative human PCSCs and activation of downstream targets such as JAK-STAT and NFKB pathways has been associated with the PCSC phenotype [45]. It appears only logical that the stroma of the tumour provides a specialized niche and influences the fate and behavior of PCSCs but the nature of the relationship and its biological consequence are poorly understood. That expression of focal adhesion and ECM-integrin signaling pathways are up-regulated in CSCs from human $\mathrm{PCa}$ as compared to the normal counterparts (both $\alpha 2 \beta 1^{\text {hi }} / \mathrm{CD} 133^{+}$), seems however to confirm these powerful reciprocal interactions [45].

Nothing is known, though, about the sequence of PCa-initiating events. Results of existing studies lead to several questions, which include distinguishing between cause and effect for the CSC and its niche. Is 'stemness' an inherent property of distinct types of cells, or acquired during a dynamic process induced and controlled by environmental cues? Can CSCs become niche-independent?
Answers to these questions are of paramount relevance for the development of potential therapeutic strategies.

\section{Therapeutic implications}

Since the work of Huggins and Hodges [56], androgen deprivation therapy (ADT) is the mainstay of management for progressive advanced PCa. Depriving PCa cells of hormonal stimuli can result in eradication of most cancer cells and so regression of androgen dependent (AD) tumours but in most cases the disease relapses and progresses to CR PCa. The molecular mechanisms underlying alterations of cell sensitivity to androgens are not well understood and several possibilities that may be responsible for evolution to the CR state have been proposed $[57,58]$. Evidence from xenograft models and clinical material indicates that the switch to $\mathrm{CR}$ growth could result from either adaptive responses to ADT and evolution of CR clones [59] or the selection/outgrowth of pre-existing (pretreatment) $C R$ and AR-independent cells. The observation that ADT can destroy secretory luminal epithelial cells while those with basal cell-like characteristics preferentially survive suggests that intrinsically resistant cells do exist in the gland and is consistent with the concept of PCSCS.

The existence of $A R^{-}$poorly differentiated clones, equipped with active mechanisms to evade irradiation or cytotoxic therapies, would offer an explanation for disease recurrence and development of the CR state. Furthermore, if PCSCs did form a dormant reservoir for tumour regrowth, ADT would have an adverse effect on disease progression and, by activating normally quiescent AR- PCSCs to repopulate the tumour, could promote CR evolution [54]. Thus, targeting of PCSCs could provide lasting, curative effects. Unfortunately, if the numbers of true PCSCs is as low as implied by the CSC hypothesis, selective eradication of these cells would be extremely difficult, if possible. Identification and inhibition of self-renewal signalling pathways such as $\mathrm{PI} 3 \mathrm{~K}$, Wnt, and Notch $[29-33,60,61]$ seem to offer an alternative and more feasible approach.

\section{Conclusions and perspectives}

While the CSC hypothesis has exciting clinical implications, there are still many unresolved issues. The most important concern is raised by identification of these unique cells, their genetic and phenotypic fingerprint, pathways and networks that control their biology, hormone sensitivity and responsiveness to paracrine environmental stimuli. The achievement of this task is hampered by a lack of suitable models. To date the gold standard to confirm CSC phenotype is serial transplantation into an animal model, where the cell recapitulates histologic heterogeneity of the original tumour. However, as human tumour cells implanted heterotopically into a normal animal niche are undoubtedly subjected to selective pressure due to incomplete immunosuppression and species- and site- specific composition of the stroma and hence its paracrine activity, this model does not precisely mimic the original human disease. Thus extrapolation from the key experimental set-up to verify CSC functional characteristics has to be treated with caution, leaving the true identity of the cell yet to be determined.

The existence of CSCs in most tumours raises little doubt about their role. The current challenge is to unravel distinguishing molecular profiles for the CSC and determine the molecular pathways of their self-renewal activity in order to target them specifically for therapeutic purposes. As there are remarkable parallels between normal stem cells and cancer stem cells, advancing knowledge of stem cell biology will have significant implications for the attempts to defeat cancer or, at least, to tame its aggressive behaviour. 


\section{REFERENCES}

1. Reya T, Morrison SJ, Clarke MF, Weissman IL: Stem cells, cancer, and cancer stem cells. Nature 2001; 414: 105-111.

2. Pardal R, Molofsky AV, He S, Morrison SJ: Stem cell self-renewal and cancer cell proliferation are regulated by common networks that balance the activation of proto-oncogenes and tumor suppressors. Cold Spring Harb Symp Quant Biol 2005; 70: 177-185.

3. Al-Hajj M, Clarke MF: Self-renewal and solid tumor stem cells. Oncogene 2004; 23: 7274-7282.

4. Passegué $\mathrm{E}$, Weisman IL: Leukemic stem cells: where do they come from? Stem Cell Rev 2005; 1: 181-188.

5. Adams JM, Kelly PN, Dakic A, et al: Role of "cancer stem cells" and cell survival in tumor development and maintenance. Cold Spring Harb Symp Quant Biol 2008; 73: 451-459.

6. Visvader J: Cells of origin in cancer Nature 2011; 469: 314-322.

7. Till JE, McCulloch EA: A direct measurement of the radiation sensitivity of normal mouse bone marrow cells. Radiation Research 1961; 14: 213-222.

8. Smith A: Embryo-derived stem cells: Of mice and men. Annu Rev Cell Dev Biol 2001; 17: 435-462.

9. Avilion AA, Nicolis SK, Pevny LH, et al: Multipotent cell lineages in early mouse development depend on SOX2 function. Genes Dev 2003; 17: 126-140.

10. Mitsui $\mathrm{K}$, Tokuzawa $\mathrm{Y}, \mathrm{Itoh} \mathrm{H}$ et al: The homeoprotein Nanog is required for maintenance of pluripotency in mouse epiblast and ES cells. Cell 2003; 113: 631-642.

11. Flores I, Blasco MA: The role of telomeres and telomerase in stem cell aging. FEBS Lett 2010; 584: 3826-3830.

12. Park IK, Morrison SJ, Clarke MF: Bmi1, stem cells, and senescence regulation. J Clin Invest 2004; 113: 175-179.

13. Burdon T, Smith A, Savatier P: Signaling, cell cycle and pluripotency in embryonic stem cells. Trends Cell Biol 2002; 12: 432-438.

14. Walsh J, Andrews PW: Expression of Wht and Notch pathway genes in a pluripotent human embryonal carcinoma cell line and embryonic stem cell. APMIS 2003; 111: 197-210.

15. Voog J, Jones DL: Stem cells and the niche: a dynamic duo. Cell Stem Cell 2010; 6: 103-115.

16. Liu AY, True LD, LaTray L, et al: Cell-cell interaction in prostate gene regulation and cytodifferentiation. Proc Natl Acad Sci USA 1997; 94: 10705-10710.

17. Abrahamsson PA, Lilja H: Partial characterization of a thyroid-stimulating hormone-like peptide in neuroendocrine cells of the human prostate gland. Prostate 1989; 14: 71-81.

18. Hayashi N, Cunha GR, Parker M: Permissive and instructive induction of adult rodent prostatic epithelium by heterotypic urogenital sinus mesenchyme. Epithelial Cell Biol 1993; 2: 66-78.

19. Hayward SW, Haughney PC, Rosen MA, et al: Interactions between adult human prostatic epithelium and rat urogenital sinus mesenchyme in a tissue recombination model. Differentiation 1998; 63: 131-140.

20. Hayward SW, Haughney PC, Lopes ES, Danielpour D, and Cunha GR: The rat prostatic epithelial cell line NRP-152 can differentiate in vivo in response to its stromal environment. Prostate 1999; 39: 205-212.

21. English HF, Santen RJ, Isaacs JT: Response of glandular versus basal rat ventral prostatic epithelial cells to androgen withdrawal and replacement. Prostate 1987; 11: 229-242.

22. Signoretti $S$, Pires $M M$, Lindauer $M_{1}$ et al: p63 regulates commitment to the prostate cell lineage. Proc Natl Acad Sci USA 2005; 102: 1135511360.

23. Tsujimura A, Koikawa $Y$, Salm, et al: Proximal location of mouse prostate epithelial stem cells: a model of prostatic homeostasis. J Cell Biol 2002; 157: 1257-1265.

24. Kurita T, Medina RT, Mills AA, Cunha GR: Role of $p 63$ and basal cells in the prostate. Development. 2004; 131: 4955-4964.

25. Collins AT, Habib FK, Maitland NJ, Neal DE: Identification and isolation of human prostate epithelial stem cells based on $\alpha 2 \beta 1$-integrin expression. J Cell Sci 2001; 114: 3865-3872.

26. Richardson $\mathrm{GD}$, Robson $\mathrm{CN}$, Lang $\mathrm{SH}$, et al: $C D 133$, a novel marker for human prostatic epithelial stem cells. J Cell Sci 2004; 117: 3539-3545.

27. Potter VR: Phenotypic diversity in experimental hepatomas: the concept of partially blocked ontogeny. The 10th Walter Hubert Lecture. Br J Cancer 1978; 38: 1-23.

28. Burkert J, Wright NA, Alison MR: Stem cells and cancer: an intimate relationship. J Pathol 2006; 209: 287-297.

29. Mulholland DJ, Xin L, Morim A, et al: Lin-Sca-1+CD49fhigh stem/progenitors are tumor-initiating cells in the Pten-null prostate cancer model. Cancer Res 2009; 69: 8555-8562.

30. Chen G, Shukeir N, Potti A, et al: Up-regulation of Wnt-1 and beta-catenin production in patients with advanced metastatic prostate carcinoma: potential pathogenetic and prognostic implications. Cancer 2004; 101: 1345-1356.

31. Reya T, Clevers H: Wnt signaling in stem cells and cancer. Nature 2005; 434: 843-850.

32. Wang $Z, L i Y$, Banerjee $S$, et al: Down-regulation of Notch-1 and Jagged-1 inhibits prostate cancer cell growth, migration and invasion, and induces apoptosis via inactivation of Akt, mTOR, and NF-kappaB signaling pathways. J Cell Biochem 2011; 112: 78-88.

33. Leong KG, Gao WQ: The Notch pathway in prostate development and cancer. Differentiation 2008; 76: 699-716.

34. Berezovska OP, Glinskii AB, Yang Z, et al: Essential role for activation of the Polycomb group $(\mathrm{PCG}$ ) protein chromatin silencing pathway in metastatic prostate cancer. Cell Cycle 2006; 5: 1886-1901.

35. Jiang L, Li J, Song L: Bmi-1, stem cel/s and cancer. Acta Biochim Biophys Sin (Shanghai) 2009; 41: 527-534.

36. Huls M, Russel FG, Masereeuw R: The role of ATP binding cassette transporters in tissue defense and organ regeneration. J Pharmacol Exp Ther 2010; 328: 3-9.

37. Storms RW, Trujillo AP, Springer JB, et al: Isolation of primitive human hematopoietic progenitors on the basis of aldehyde dehydrogenase activity. Proc Natl Acad Sci USA 1999; 96: 9118-9123.

38. McDonald SA, Graham TA, Schier $S$, et al: Stem cells and solid cancers. Virchows Arch 2009; 455: 1-13.

39. http://info.cancerresearchuk.org/cancerstats/types/prostate/incidence/

40. Moul JW, Srivastava S, McLeod DG: Molecular implications of the antiandrogen withdrawal syndrome. Semin Urol 1995; 13: 157-163.

41. Liu AY, Nelson PS, van den Engh G, Hood L: Human prostate epithelial cell-type cDNA libraries and prostate expression patterns. Prostate 2002; 50: 92-103.

42. Collins AT, Berry PA, Hyde $C$, et al: Prospective identification of tumorigenic prostate cancer stem cells. Cancer Res 2005; 65: 10946-10951.

43. Patrawala L, Calhoun T, Schneider-Broussard R, et al: Highly purified CD44+ prostate cancer cells from xenograft human tumors are enriched in tumorigenic and metastatic progenitor cells. Oncogene 2006; 25: 1696-1708.

44. Gu G, Yuan J, Wills ML, Kasper S: Prostate cancer cells with stem cell characteristics reconstitute the original human tumor in vivo. Cancer Res 2007; 67: 4807-4815.

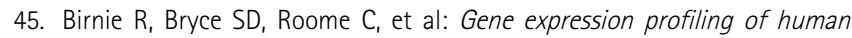
prostate cancer stem cells reveals a pro-inflammatory phenotype and the importance of extracellular matrix interactions. Genome Biol 2008; 9: R83.

46. Wang $X$, Kruithof-de Julio $M$, Economides KD, et al: A luminal epithelial stem cell that is a cell of origin for prostate cancer. Nature 2009; 461: 495-500.

47. Maitland NJ, Frame FM, Polson ES, et al: Prostate cancer stem cells: do they have a Basal or luminal phenotype? Horm Cancer 2011; 2: 47-61.

48. Maitland NJ: Pathobiology of the human prostate. Trends Urol Gynaecol Sex Health 2008; 13: 12-19.

49. Lawson DA, Zong $Y$, Memarzadeh $S$, et al: Basal epithelial stem cells are efficient targets for prostate cancer initiation. Proc Natl Acad Sci USA 2010; 107: 2610-2515. 
50. Goldstein AS, Huang J, Guo C, et al: Identification of a cell of origin for human prostate cancer. Science 2010; 329: 568-571.

51. Olumi AF, Grossfeld GD, Hayward SW, et al: Carcinoma-associated fibroblasts direct tumor progression of initiated human prostatic epithelium. Cancer Res 1999; 59: 5002-5011.

52. Thalmann GN, Rhee $\mathrm{H}$, Sikes RA, et al: Human Prostate Fibroblasts Induce Growth and Confer Castration Resistance and Metastatic Potential in LNCaP Cells. Eur Urol 2010; 58: 162-171.

53. Sung SY, Hsieh $\mathrm{CL}$, Law $\mathrm{A}$, et al: Coevolution of prostate cancer and bone stroma in three-dimensional coculture: implications for cancer growth and metastasis. Cancer Res 2008; 68: 9996-10003.

54. Lang SH, Frame FM, Collins AT: Prostate cancer stem cells. J Pathol 2009; 217: 299-306.

55. Kucia $M$, Jankowski $K$, Reca $R$, et al: CXCR4-SDF-1 signaling, locomotion, chemotaxis and adhesion. J Mol Histol 2004; 35: 233-245.

56. Huggins $C_{1}$ Hodges CV: Studies on prostatic cancer. I. The effect of castration, of estrogen and androgen injection on serum phosphatases in metastatic carcinoma of the prostate. CA Cancer J Clin 1972; 22: 232-240.

57. Taplin ME, Bubley GJ, Shuster TD, et al: Mutation of the androgen-receptor gene in metastatic androgen-independent prostate cancer. N Engl J Med 1995; 332: 1393-1398.

58. Barton J, Blackledge $\mathrm{G}$, Wakeling A: Growth factors and their receptors: new targets for prostate cancer therapy. Urology 2001; 58 (2 Suppl 1): 114-122.

59. Craft N, Chhor C, Tran C, et al: Evidence for clonal outgrowth of androgenindependent prostate cancer cells from androgen-dependent tumors through a two-step process. Cancer Res 1999; 59: 5030-5036.
60. Glinsky GV, Berezovska O, Glinskii AB: Microarray analysis identifies a deathfrom-cancer signature predicting therapy failure in patients with multiple types of cancer. J Clin Invest 2005; 115: 1503-1521.

61. Goldstein AS, Stoyanova T, Witte ON: Primitive origins of prostate cancer: in vivo evidence for prostate-regenerating cells and prostate cancer-initiating cells. Mol Oncol 2010; 4: 385-396.

\section{Correspondence}

Hanna Romańska-Knight

Department of Molecular Pathology and Neuropathology

Medical University of Łódź

251, Pomorska Street

92-216 Łódź, Poland

phone: +42 2725611

hanna.romanska@gmail.com 